\title{
Patrones de diversidad alfa en tres fragmentos de bosques montanos en la región norte de los Andes, Colombia
}

\author{
Wilson López ${ }^{1} \&$ Álvaro Duque ${ }^{2}$ \\ 1. Maestría en bosques y conservación ambiental, Universidad Nacional de Colombia, Sede Medellín, Colombia; \\ ewlopez@unalmed.edu.co \\ 2. Departamento de ciencias forestales, Universidad Nacional de Colombia, Sede Medellín, Colombia; \\ ajduque@unalmed.edu.co
}

Recibido 04-V-2009. Corregido 21-IX-2009. Aceptado 21-X-2009.

\begin{abstract}
Alpha diversity patterns in three fragmented forests of Northern Andes, Colombia. Diversity studies in forests are useful for conservation management. We analyzed the patterns of alpha diversity in three protected forests along an altitudinal gradient in the Northern part of the Central Mountain Range, Colombia. The study was based on six permanent plots of 1 ha (two per area). According to plant size, two main categories were used: canopy (diameter at breast height $\geq 10 \mathrm{~cm})$ and understory $(1 \mathrm{~cm} \leq$ diameter at breast height $<10 \mathrm{~cm})$. Species-area curves showed differences in species richness by size category in all regions. Instead, speciesindividual curves were independent of the size in Anorí and Angelópolis, but not in Belmira. Diversity patterns, as measured by Fisher's alpha index, showed a strong tendency to reach a plateau for sample sizes larger than 1000 individuals. Neither species-area curves nor species-individual curves showed an asymptote; however, the species accumulation rate in Belmira was very low (0.05) when compared to Anorí and Angelópolis (0.25). Fisher's alpha index is a very useful tool to understand the extent of relative differences in diversity among regions or forest types along altitudinal gradients, even when sample size is relatively small. Our results support dispersal limitation as the main mechanism structuring species assemblages at very fine scales, and the monotonic increment of density-dependence processes determining the tree community structure at larger spatial scales. Likewise, we found that density-dependence tends to increase with altitude. Rev. Biol. Trop. 58 (1): $483-$ 498. Epub 2010 March 01.
\end{abstract}

Key words: mountain forests, Andes, alpha diversity, species-area curves, species-individual-curves, Fisher's alpha, dispersal limitation, density-dependence, Colombia.

Las curvas de especies por área han sido uno de los más antiguos y relevantes paradigmas en ecología y conservación (McArtur \& Wilson 1967, Harte et al. 1999, 2009, Bordade-Água et al. 2002). Las curvas de especiesárea han sido utilizadas para definir el área o tamaño mínimo de muestra (Rosenzweig 1995), así como para identificar los mecanismos determinantes de la coexistencia de especies, entre otros usos (Condit et al. 1996, He \& Legendre 2002). En el primer caso, se asume que una vez que se alcanza una asíntota en el número de especies, el tamaño de muestra correspondiente es el área mínima de muestreo, que contiene una muestra representativa de la comunidad (Rosenzweig 1995). En el segundo caso, la comparación de las pendientes entre curvas y/o con los valores esperados por azar (Hurberlt 1971), permite inferir acerca del papel de los procesos denso dependientes, como el aumento de mortalidad de conespecíficos por cercanía con el parental (Janzen 1970, Connell 1971), o de factores biológicos, como la limitación en dispersión (Hubell 2001) y el 
patrón de distribución agregado de las especies, en el control de los ensamblajes de especies en las comunidades vegetales.

Es ampliamente aceptado que la relación entre el número de especies $S$ y el área $A$ es una función de potencia de la forma $S=c A^{z}$ (McArtur \& Wilson 1967, Rosenzweig 1995). El valor de $z$ denota la tasa a la cual se acumulan las especies a medida que aumenta el área, y su magnitud y variación han sido objeto de debate (Rosenzweig 1995, Harte et al. 1999, Borda-de-Agua et al. 2002, Harte et al. 2009). Los rangos teóricos planteados para $z$ varían desde aproximadamente 0.25 (Preston 1962) hasta 0.11 (Condit et al. 1996), pasando por valores intermedios alrededor de 0.16 y 0.12 como los propuestos por Harte et al. (2008) y Rosenzweig (1995), respectivamente. Recientes avances teóricos apoyados en la teoría de la información, han propuesto un modelo universal donde el valor de $z$ tiende a cero, lo cual implica la existencia de una asíntota para el número de especies en algún punto del espacio (Harte et al. 2009). Esta visión contrasta con lo expuesto en la teoría neutral (Hubbell 2001), donde se espera un incremento continuo de especies producto del balance entre los procesos de especiación y extinción que se dan a escala regional y de paisaje (Zillio \& Condit 2007).

Sin embargo, la pendiente de la curva de especies-área definida por el valor de $z$ ha mostrado ser altamente dependiente de la escala espacial (He \& Legendre 1996, Plotkin et al. 2000). A escala local, en una gráfica log-log, la curva especies-área aún es curvilínea, lo cual dificulta su uso para extrapolaciones dado su carácter no lineal, y por tanto, su tasa de acumulación de especies variable (Condit et al. 1996, 1998; Borda-de-Agua et al. 2002). Por este motivo, y dependiendo del número mínimo de individuos considerados en la muestra, algunos autores sugieren como una medida más apropiada para estudiar la diversidad, los análisis basados en la relación especies-individuos o índices que involucran la abundancia relativa, en vez de la relación especies-área (Gotelli \& Colwell 2001, Condit et al. 1996). En estudios de diversidad arbórea, los índices de diversidad que han mostrado mayor estabilidad con respecto al tamaño de la muestra han sido el alfa de Fisher y el de Shannon-Wiener (Magurran 1988, Condit et al. 1996, Rosenzweig 1995). Sin embargo, dado que el alfa de Fisher cuantifica e involucra intrínsecamente la relación entre el número de individuos y el número de especies presentes, ha sido efectivamente empleado para estimar la riqueza de especies en áreas geográficas extensas usando muestras provenientes de áreas reducidas (Condit et al. 1996, 2005, Hubbell 2001).

El presente estudio, fue llevado a cabo en áreas de reserva de bosques montanos en el extremo norte de la cordillera Central en Colombia usando información proveniente de seis parcelas permanentes de una hectárea, tuvo como objetivo principal evaluar las generalizaciones reportadas por investigaciones previas en bosques tropicales de tierras bajas (Condit et al. 1996) en cuanto a los mecanismos y procesos que determinan la variación de la diversidad y las tasas de acumulación de especies arbóreas. Estudios sobre patrones de diversidad de especies en ecosistemas de montaña han mostrado la existencia de un gradiente de diversidad que varía negativamente con la altitud (Lieberman et al. 1996, Boyle 1996, Gentry 1995, Hemp 2005). Por este motivo, se esperaría que en bosques de alta montaña como los aquí considerados, el valor de $z$ se estabilice a tamaños de muestra menores que los encontrados o reportados para tierras bajas (Condit et al. 1996, 1998), y que la densodependencia disminuya inversamente con la altitud. Los objetivos centrales buscan responder las siguientes preguntas: ¿Cuáles son las diferencias al evaluar los patrones de riqueza, para dos categorías de tamaño, en función del área y el número de individuos muestreados? ¿Cómo son los patrones de diversidad alfa en cada una de las regiones? y ¿Qué procesos podrían estar determinando dichos patrones? El estudio espera aportar herramientas y oportunidades de manejo de estas áreas de bosque destinadas a la conservación. 


\section{MÉTODOS}

Sitio de estudio: El estudio fue llevado a cabo en bosques pertenecientes a tres áreas de reserva regionales ubicadas en el extremo norte de la cordillera Central de los Andes en Colombia, departamento de Antioquia. La Reserva Forestal La Forzosa, localizada en la región de Anorí $\left(6^{\circ} 58^{\prime} \mathrm{N}, 75^{\circ} 09^{\prime} \mathrm{W}\right)$ a una altitud de $1750 \mathrm{msnm}$, se ubica en la zona de vida bosque muy húmedo premontano (bmh-PM), según el sistema de clasificación de zonas de vida de Holdridge (1996), la reserva aún permanece cubierta en un $77 \%$ por bosques desde muy perturbados (rastrojos altos) hasta bosques primarios con bajo grado de intervención (Gutiérrez-C. 2002). La reserva El Romeral, localizada en la región de Angelópolis (6 ${ }^{\circ} 9.5^{\prime}$ $\left.\mathrm{N}, 75^{\circ} 42^{\prime} \mathrm{W}\right)$ a una altitud de $2100 \mathrm{msnm}$ está conformada por bosques naturales en diferentes estados de sucesión con predominio de bosques naturales primarios con bajo grado de intervención; se ubica en la zona de vida bosque muy húmedo montano bajo (bmh-MB) y por último el Área de Manejo Especial Sistema de Páramos y Bosques Alto Andinos en la región de Belmira $\left(6^{\circ} 35^{\prime} \mathrm{N}, 75^{\circ} 32^{\prime} \mathrm{W}\right)$ ubicados a una altitud aproximada de $2900 \mathrm{msnm}$ dentro de la zona de vida bosque muy húmedo montano (bmh-M); esta región se caracteriza por poseer un paisaje bastante fragmentado con importantes asociaciones de robledales (Quercus humboldtii Bonpl.).

En los tres bosques estudiados se asumen evidencias de intervención humana producto de la fragmentación y extracción selectiva en el pasado de algunas especies, lo cual podría haber afectado indirectamente las características estructurales de los bosques, aunque los procesos de intervención no están bien documentados (Duque et al. 2008).

Los criterios tomados en cuenta para la selección de las parcelas se dirigieron primeramente a la disponibilidad de fragmentos de bosque más o menos bien conservados sin signos de intervención importante en los últimos 40 o 50 años, que tuvieran el respaldo de estar dentro de predios que brinden un soporte jurídico para su protección, y finalmente la facilidad de acceso. Las áreas seleccionadas cumplen además con requisitos importantes como: 1) Área total de bosque mayor de 300ha. 2) Relativa buena accesibilidad. 3) Uso actual y potencial dedicado a la conservación. 4) Poseen vigilancia o control. 4) En cada región las parcelas fueron ubicadas de manera aleatoria.

Método de muestreo: El protocolo de muestreo fue exactamente igual para las tres regiones. En cada región se realizó el montaje de dos parcelas permanentes de una hectárea de forma cuadrada $(100 \mathrm{mx} 100 \mathrm{~m})$. En cada parcela se censaron todos los individuos arbóreos, incluyendo palmas y helechos arbóreos, con diámetro a la altura del pecho (DAP) $\geq 10 \mathrm{~cm}$; a su vez, en cada parcela de una hectárea se ubicó una subparcela de $40 \mathrm{mx} 40 \mathrm{~m}$ donde se censó la vegetación leñosa entre $1 \mathrm{~cm} \leq \mathrm{DAP}<10 \mathrm{~cm}$, para los mismos hábitos de crecimiento antes señalados los cuales incluyen la regeneración de las especies del dosel y la vegetación leñosa propia del sotobosque incluyendo palmas y helechos arbóreos.

Se realizó la colección botánica de todas las especies en las seis parcelas. La identificación se realizó por comparación directa en los herbarios del Herbario Gabriel Gutiérrez V. (MEDEL) y Herbario Universidad de Antioquia (HUA), la consulta de claves y la utilización de información de herbarios virtuales: Herbario Nacional Colombiano (COL), Herbario del Jardín Botánico Missouri (MO), Jardín Botánico de Nueva York (NY). Se siguió la nomenclatura de familias y géneros de Mabberley (1989). Las especies no identificadas plenamente fueron definidas en morfotipos, los cuales fueron tratados como especies cuando se realizaron los análisis. Las variedades y subespecies fueron tomadas en cuenta como especies independientes. La nomenclatura botánica y sinonimia fue revisada en la Base de Datos de Botánica (IPNI) y la base de datos del MO. El nivel de identificación fue de $92.6 \%$ en la categoría de familia y $60 \%$ en la de especies. Estos datos están basados en 2758 colecciones de plantas. 
Curvas especies-área y especies-individuos: Para el análisis, cada parcela fue dividida en cuadrantes no traslapados, en los cuales se hizo el conteo del número de especies e individuos para generar las curvas de acumulación de especies en dos clases diamétricas: individuos con $\mathrm{DAP} \geq 10 \mathrm{~cm}$ los cuales, para efectos prácticos, de aquí en adelante se denominarán individuos de dosel; e individuos con $1 \mathrm{~cm} \leq \mathrm{DAP}<10 \mathrm{~cm}$ o individuos de sotobosque. Para los individuos del dosel los cuadrantes fueron de $10 \mathrm{mx} 10 \mathrm{~m}(\mathrm{n}=200), 20 \mathrm{~m} \times 20 \mathrm{~m}(\mathrm{n}=50)$, $50 \mathrm{mx} 50 \mathrm{~m}(\mathrm{n}=8)$ y $100 \mathrm{~m} \times 100 \mathrm{~m}(\mathrm{n}=2)$. Para los individuos del sotobosque de $10 \mathrm{mx} 10 \mathrm{~m}(\mathrm{n}=32)$, $20 m \times 20 m(n=8)$ y $40 m x 40 m(n=2)$. Las curvas de acumulación de especies se construyeron de dos formas: primero usando las áreas asociadas con los tamaños de cuadrante arriba mencionados. Segundo, empleando el número promedio de individuos referido a cada tamaño de cuadrante (Gotelli \& Colwell 2001). Además se construyeron las curvas de acumulación total por región, en términos del área e individuos. Para los cálculos se empleó el programa EstimateS versión 7.5.1 (Colwell 2005), opción muestra sin reemplazo, con 200 iteraciones para cada tamaño de cuadrante y categoría de tamaño.

Estimador de riqueza: La riqueza de especies observada en tres diferentes tamaños de cuadrante $(10 \mathrm{mx} 10 \mathrm{~m}, 20 \mathrm{mx} 20 \mathrm{~m}$ y $40 \mathrm{mx} 40 \mathrm{~m})$, se comparó con la riqueza esperada estimada con base en la ecuación de Hurlbert (1971).

El método analítico de Hurlbert (1971) estima el número de especies con base en submuestras de individuos seleccionados de manera aleatoria. Dado un conjunto de abundancias $\mathrm{N}_{\mathrm{i}}$ para $\mathrm{S}$ especies, siendo $\mathrm{N}=\Sigma \mathrm{N}_{\mathrm{i}}$ el número total de individuos, el número de $s$ especies en una submuetra de $n$ individuos tomados al azar es $\mathrm{E}(\mathrm{s})=\Sigma \mathrm{p}_{\mathrm{i}}\left(\mathrm{N}_{\mathrm{i}}, \mathrm{N}, \mathrm{n}\right)$ donde $\mathrm{pi}(\mathrm{Ni}, \mathrm{N}$, n) es la probabilidad de encontrar i especies en la submuestra (Hurlbert 1971), la cual se halla mediante la fórmula hipergeométrica.

$$
p_{i}\left(N_{i}, N, n\right)=1-\left[\left(\frac{N-N_{i}}{N}\right)\left(\frac{N-N_{i}-1}{N}\right)\left(\frac{N-N_{i}-2}{N}\right) \cdots\left(\frac{N-N_{i}-n-1}{N}\right)\right]
$$

Para esto se desarrolló un programa en Fortran bajo Windows para el cálculo del valor esperado de especies para un número de individuos.

Valor z: Se graficó el cambio en la tasa de acumulación de especies (valor $z$ ) respecto al número de individuos mediante la relación

$$
z=\frac{\left(\log S_{i+1}-\log S_{i}\right)}{\left(\log N_{i+1}-\log N_{i}\right)}
$$

donde $S_{i}$ y $N_{i}$ son el número de especies e individuos por cuadrante $i$ de $10 \mathrm{mx} 10 \mathrm{~m}$.

Diversidad: Para estimar la diversidad arbórea se empleó el índice alfa de Fisher, definido por la fórmula:

$$
S=\alpha \ln \left(1+\frac{n}{\alpha}\right)
$$

Donde $S$ es el número de especies, $n$ el número de individuos y $\alpha$ el alfa de Fisher. Al igual que las curvas de acumulación de especies respecto al área y el número de individuos, se construyeron curvas de acumulación del índice alfa de Fisher frente al número de individuos para las dos categorías de tamaño (dosel y sotobosque). Para esto se utilizó el programa EstimateS versión 7.5.1 (Colwell 2005). Por interpolación lineal se halló el valor del índice para exactamente 1000 individuos para cada una de las regiones, además se calculó el valor del índice para cada parcela y el promedio para una hectárea. El valor del alfa de Fisher para una hectárea fue el valor promedio de los valores en cada parcela.

Por último, para poder comparar las diferencias en términos de la riqueza de especies y la diversidad entre las regiones estudiadas, se graficó la razón o cociente tanto de la riqueza como de la diversidad a un número constante 
de individuos para cada una de las regiones. De esta manera es posible interpretar cuál es la magnitud y tendencia de las diferencias de forma comparativa entre cada uno de los bosques considerados.

\section{RESULTADOS}

La mayor dominancia para las especies del dosel se presentó en la región de Belmira [Quercus humboldtii y Tibouchina lepidota (Bonpl.) Baill. con el 55\% y $6 \%$ de los individuos respectivamente], seguida por Angelópolis (Croton smithianus Croizat y Matudaea colombiana Lozano con el 14\% y $8 \%$ de los individuos) y por último la región de Anorí (Compsoneura sp. y Q. humboldtii con 6\% y $5 \%$ de los individuos). Las especies más abundantes en el sotobosque, en la región de Belmira fueron Miconia resima Naudin y $Q$. humboldtii con el $10 \%$ de los individuos cada una), la primera es exclusiva del sotobosque y la segunda es regeneración natural de un representante del dosel; en Angelópolis Allomaieta cf. hirsuta (Gleason) Lozano y Palicourea angustifolia Kunth con 16\% y 7\% respectivamente, fueron las más abundantes siendo exclusivas del sotobosque; y en Anorí las especies Tovomita weddelliana Planch. \& Triana y Macrolobium costarricense W.C. Burger con $5 \%$ y $4 \%$ respectivamente, ambas regeneración de especies del dosel.

El $41 \%, 46 \%$ y $60 \%$ de las especies del dosel en Anori, Angelópolis y Belmira respectivamente, están representadas en el sotobosque. Los porcentajes restantes corresponden a especies exclusivas del sotobosque y regeneración de otras especies arbóreas de dosel que no entraron en el inventario. Una gran proporción de las especies (50\%) pueden ser catalogadas como localmente raras las cuales presentaron tres o menos individuos en todo el inventario (López 2008).

Un total de 28 especies fueron reportadas con algún grado de riesgo o amenaza de acuerdo con las listas rojas de especies amenazadas de la IUCN (2008). Las especies que presentaron la mayor calificación en términos de amenaza fueron Magnolia espinalii (Lozano) Govaerts encontrada en la región de Angelópolis y $M$. yarumalensis (Lozano) Govaerts encontrada en la región de Anorí, las cuales están en categoría de críticamente amenazadas (CR) debido a su nivel de endemismo y a la reducción del hábitat, fuertemente fragmentado en la región Andina colombiana (Rodríguez et al. 2004).

Curvas especies-área: La forma de las curvas especies-área fue similar en los tres bosques. En escala logarítmica las curvas fueron casi lineales con una leve concavidad hacia abajo; en todos los casos, fueron diferentes y no paralelas entre las dos clases de tamaño. La tasa inicial de acumulación de especies con respecto al área fue mucho mayor en las especies de sotobosque (Fig. 1, A, B, C). Cuando se analizó la forma de la curva de acuerdo con el tamaño de cuadrante $(10 \mathrm{mx} 10 \mathrm{~m}, 20 \mathrm{mx} 20 \mathrm{~m}, 40 \mathrm{mx} 40 \mathrm{~m}$ y $50 \mathrm{~m} \times 50 \mathrm{~m}$ ), en escala logarítmica, no hubo diferencias entre ellas (no se presenta este resultado). Sin embargo, a medida que aumentó el área, las curvas tienden a converger, lo cual muestra un incremento en la proporción de las especies totales que caen en los diámetros superiores cuando se incrementa el área. Por ello la relación entre el número de especies entre el sotobosque y el dosel es máxima en el tamaño de muestra más pequeño. Cuando se analizaron en conjunto todos los datos por región, se observan curvas con una leve concavidad hacia abajo. Las curvas de Anorí y Angelópolis mostraron un comportamiento similar, con valores de riqueza en términos del área muy diferentes y superiores a Belmira (Fig. 1D).

Curvas especies-individuos: En contraste, y con excepción de lo encontrado en Belmira, las curvas especies-individuos fueron idénticas para las dos categorías de tamaño (Fig. 2 A, B, C). Este resultado significa que el número de especies en un número de individuos dado fue prácticamente independiente de la categoría de tamaño. En el caso de la región de Belmira, y similar a lo encontrado con respecto al área, las curvas generadas al considerar las especies del sotobosque estuvieron levemente por encima 

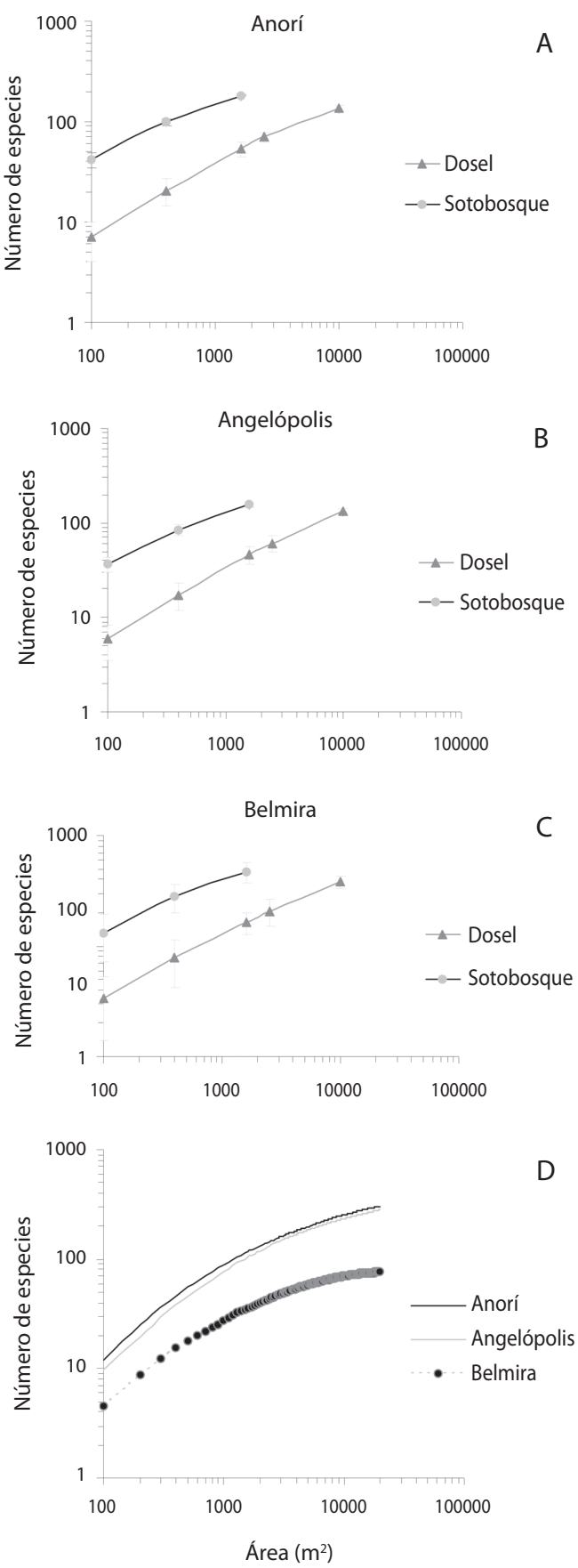

Fig. 1. Curvas especies-área en escala logarítmica para tres regiones y dos diferentes categorías de tamaño. A, B y C corresponden a los promedios de especies por cuadrantes de $10 \mathrm{mx} 10 \mathrm{~m}, 20 \mathrm{mx} 20 \mathrm{~m}, 40 \mathrm{mx} 40 \mathrm{~m}, 50 \mathrm{mx} 50 \mathrm{~m}$ y $100 \mathrm{mx} 100 \mathrm{~m}$. D corresponde a las curvas de acumulación por región considerando en conjunto los datos de las dos categorías de tamaño (dosel y sotobosque). de las curvas obtenidas con las especies del dosel; es decir, se encontró una mayor acumulación de especies a medida que aumentó el número de individuos en el sotobosque que en el dosel, develando la mayor dominancia y abundancia de unas pocas especies en el dosel tales como Q. humboldtii Bonpl. A nivel regional la forma de las curvas define claramente la relación negativa planteada entre la diversidad y la altitud (Fig. 2D) (Lieberman et al. 1996, Boyle 1996, Gentry 1995, Hemp 2005).

Valor z: La pendiente de las curvas especies-individuos (valor $z$ o tasa de acumulación de especies) declinó de forma constante con el aumento del número de individuos en las tres regiones (Fig. 3), alcanzando valores diferentes. Cuando se consideró el comportamiento de $z$ con respecto al número de individuos en relación con el tamaño de los individuos (dosel y sotobosque), los resultados de las tres regiones fueron diferentes. En Anorí la tasa de acumulación de especies disminuyó más rápidamente para las especies del sotobosque, mientras que en Angelópolis disminuyo más rápidamente para las especies del dosel, pero en ambas regiones la disminución fue casi lineal y de forma paralela entre las dos categorías de tamaño. Por su parte en Belmira la tasa de disminución fue mayor para el sotobosque que para el dosel pero a diferencia de las anteriores la disminución no fue lineal ni paralela entre las dos categorías de tamaño (Fig. 3 A, B, C). $\mathrm{Al}$ analizar todos los datos de cada región en conjunto, las curvas en la región de Anorí y Angelópolis fueron casi lineales a partir de 500 individuos llegando a valores de 0.25 en 3800 individuos. Por su parte en Belmira la curva claramente sigue decreciendo llegando a valores muy bajos $(0.05)$ con la tendencia a

Fig. 1. Species area curves on logarithmic scale (log-log) for three regions and two d.b.h size. A, B and C belong to average values by quadrats of $10 \mathrm{mx} 10 \mathrm{~m}, 20 \mathrm{mx} 20 \mathrm{~m}$, $40 \mathrm{mx} 40 \mathrm{~m}, 50 \mathrm{mx} 50 \mathrm{~m}$ and $100 \mathrm{mx} 100 \mathrm{~m}$. D: belong to the species accumulation curves by region, considering all the size categories data (canopy and understory). 

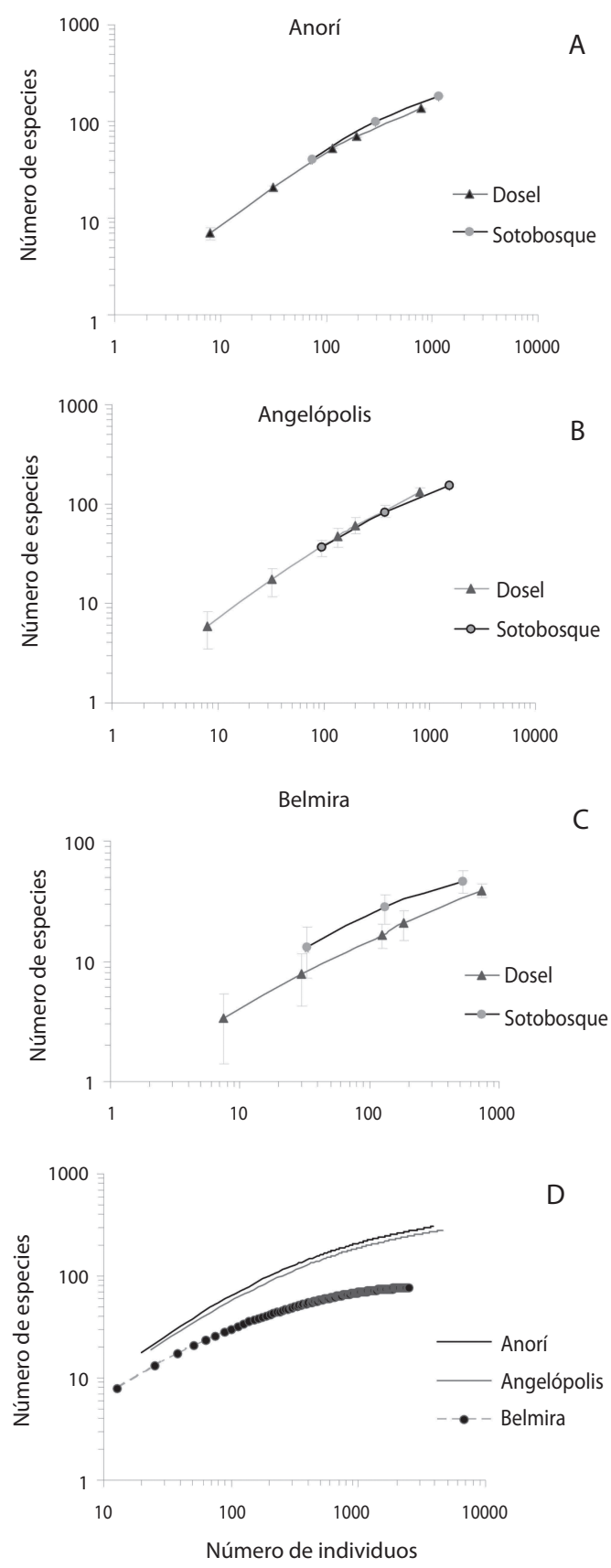

Fig. 2. Curvas especies-individuos en escala logarítmica para tres regiones y dos diferentes categorías de tamaño. A, B y C corresponden a las curvas de acumulación con base en los promedios de las especies e individuos en cuadrantes de $10 \mathrm{mx} 10 \mathrm{~m}, 20 \mathrm{~m} \times 20 \mathrm{~m}, 40 \mathrm{mx} 40 \mathrm{~m}, 50 \mathrm{mx} 50 \mathrm{~m}$ y $100 \mathrm{mx} 100 \mathrm{~m}$. alcanzar un valor asintótico entre 2500 y 3000 individuos (Fig. 3 D).

Diferencias de riqueza y diversidad entre regiones: En las tres regiones hubo menos especies en parcelas cuadradas que las esperadas a partir de colecciones aleatorias de individuos por la fórmula de Hurlbert (Cuadro 1). En Anorí, sin embargo, se tuvo la más baja sobre-estimación de la riqueza observada (entre el 9\% y $21 \%$ ), siendo mínima en los cuadrantes de mayor tamaño. Esto sugiere una distribución cuasi aleatoria de las especies del dosel y sotobosque (menor grado de gregarismo). En Angelópolis, la sobre-estimación estuvo entre el $19 \%$ y $32 \%$, siendo mínima en los cuadrantes de mayor tamaño para las especies del sotobosque. Por su parte, en Belmira la sobre-estimación estuvo entre el $27 \%$ y $104 \%$, siendo máxima para las especies del dosel y sotobosque en los cuadrantes de tamaño pequeño.

Diversidad: La diversidad, medida con el índice alfa de Fisher, se incrementó con el número de individuos en las tres regiones y fue diferente en las categorías de tamaño (Fig. 4 A, B, C). No obstante, hubo diferencias entre las regiones. En Anorí la diversidad siempre fue mayor para el dosel que para el sotobosque, mientras en Angelópolis esta tiende a igualarse entre las dos categorías de tamaño a partir de 2000 individuos. Por su parte en Belmira la diversidad fue mayor para el sotobosque.

En todas las regiones las curvas muestran un rápido incremento en muestras menores a 800 individuos, y a partir de este número el incremento es más lento. Sin embargo, cuando se consideraron todos los datos en conjunto por

Fig. 2. Species individual curve on logarithmic scale (log-log) for three regions and two d.b.h size. A, B and C belong to the species accumulation curves, based in the species and individual averages of $10 \mathrm{mx} 10 \mathrm{~m}, 20 \mathrm{mx} 20 \mathrm{~m}$, $40 \mathrm{~m} \times 40 \mathrm{~m}, 50 \mathrm{mx} 50 \mathrm{~m}$ and $100 \mathrm{~m} \times 100 \mathrm{~m}$ quadrats. 

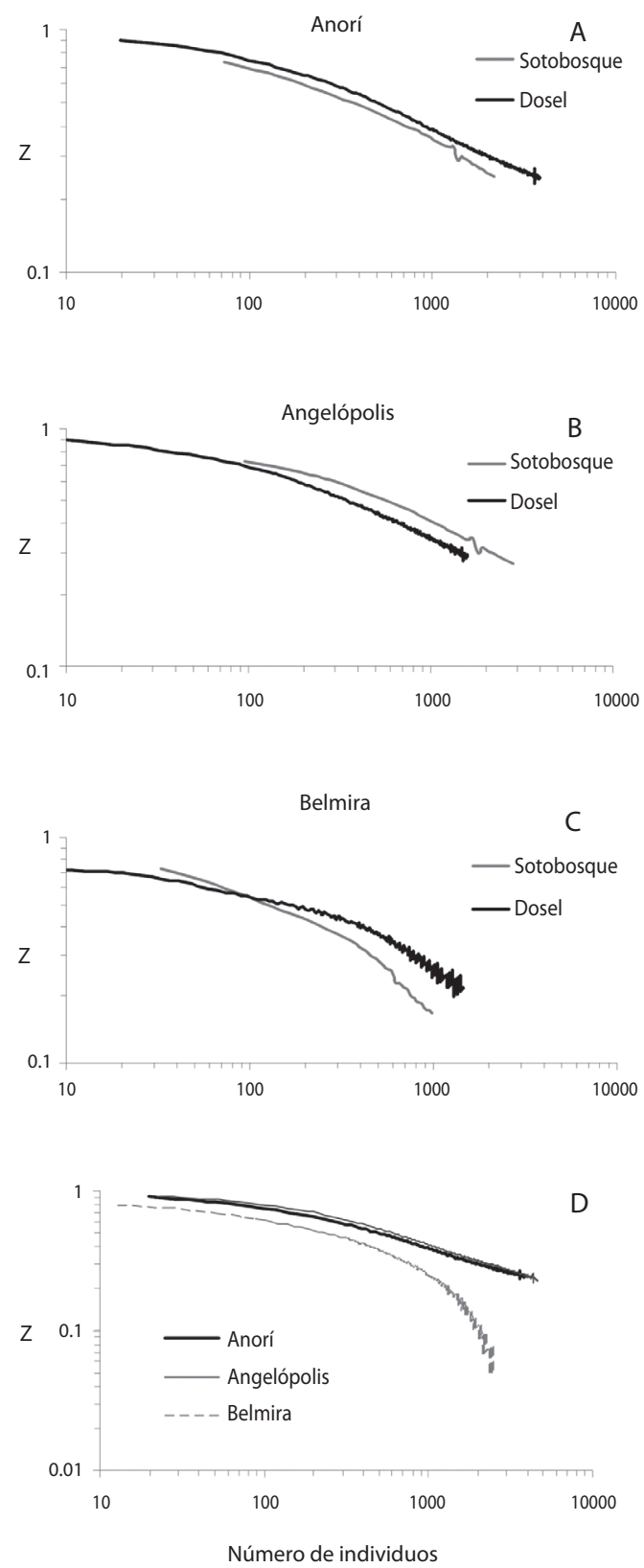

Fig. 3. Pendiente de las curvas especies-individuos con transformación logarítmica (valores z) graficado contra el número de individuos. Las pendientes fueron calculadas separadamente para dosel (puntos negros) y sotobosque (puntos en gris) para cada región (A, B y C) y por región con los datos de dosel y sotobosque en conjunto (D). región (Fig. 4D), en Belmira el valor del índice aumenta hasta 700 individuos, a partir de allí y hasta 1600 individuos el índice es constante y a partir de 1600 individuos el índice tiende a disminuir; mientras que en Anorí el índice alcanza un valor constante a partir de 1000 individuos, en Angelópolis se alcanza a partir de 2500 individuos (Fig. 4 D). Los valores de diversidad promedio por hectárea y a un número fijo de 1000 individuos, medidos con el índice alfa de Fisher, fueron por mucho mayores en Anorí y Angelópolis que en Belmira (Cuadro 2).

Riqueza relativa: La riqueza relativa de especies entre regiones presentó dos tendencias: primero, fue casi constante entre Anorí y Angelópolis a partir de una muestra de 1800 individuos siendo Anorí 1.13 veces más rico en especies que Angelópolis. Segundo, fue creciente entre Belmira y las otras dos regiones (Fig. 5). Esta última tendencia se debe, básicamente, a que la tasa de acumulación de especies en la región de Belmira a partir de cierto número de individuos es mucho más baja que en las otras dos regiones $\mathrm{y}$, por tanto, a medida que se incrementa el muestreo las diferencias en riqueza de especies se hacen cada vez mayores. Con una muestra de 2700 individuos, Anorí y Angelópolis fueron 3.57 y 3.15 veces, respectivamente, más ricos en especies que Belmira, con tendencia a seguir aumentando.

La diversidad relativa con base en el índice alfa de Fisher presentó un comportamiento similar al encontrado por la riqueza de especies, con la gran diferencia que el cociente entre diversidades a un número dado de individuos mostró menos variación que para la riqueza,

Fig. 3. Slopes ( $z$ values) of log-log species individual curves. The slopes were calculate separately to canopy species (black dots) and to understory species (grey dots) for every region $(\mathrm{A}, \mathrm{B}, \mathrm{C})$ and for each region with the canopy and understory data (D). 


\section{CUADRO 1}

Número de especies observadas (S observado) y esperadas [E(S)] para tres tamaños de cuadrantes y dos categorias de tamaño en cada una de las regiones. El número esperado de especies fue calculado con la fórmula de Hurlbert para un número dado de individuos. El número de individuos y especies observadas corresponde al valor promedio para cada tamaño de cuadrante.

\section{TABLE 1}

Observed (S observado) and expected [E(s)] species number for some quadrat sizes and two d.b.h. class for Anori, Angelópolis and Belmira region. Expected number of species was calculated by the Hurlbert's formula. The number of individuals and species observed was the average value of each plot size.

\begin{tabular}{|c|c|c|c|c|c|}
\hline Tamaño de cuadrante & $\mathrm{DAP}(\mathrm{cm})$ & No individuos & E(S) Hurlbert & S observado & $\%$ sobrestimación \\
\hline \multicolumn{6}{|c|}{ Anorí } \\
\hline \multirow[t]{2}{*}{$10 \times 10$} & $1 \leq \mathrm{DAP}<10$ & 72.5 & 50.2 & 41.4 & 21.3 \\
\hline & $\geq 10$ & 7.9 & 7.5 & 6.8 & 10.5 \\
\hline \multirow[t]{2}{*}{$20 \times 20$} & $1 \leq \mathrm{DAP}<10$ & 290.0 & 114.9 & 99.1 & 15.9 \\
\hline & $\geq 10$ & 31.3 & 24.0 & 20.8 & 15.2 \\
\hline \multirow[t]{2}{*}{$40 \times 40$} & $1 \leq \mathrm{DAP}<10$ & 1160.5 & 199.0 & 183.0 & 8.7 \\
\hline & $\geq 10$ & 114.875 & 59.8 & 53.5 & 11.8 \\
\hline \multicolumn{6}{|c|}{ Angelópolis } \\
\hline \multirow[t]{2}{*}{$10 \times 10$} & $1 \leq \mathrm{DAP}<10$ & 94.8 & 48.8 & 37.0 & 32.0 \\
\hline & $\geq 10$ & 8.0 & 7.1 & 6.0 & 19.0 \\
\hline \multirow[t]{2}{*}{$20 \times 20$} & $1 \leq \mathrm{DAP}<10$ & 379.4 & 109.1 & 84.1 & 29.7 \\
\hline & $\geq 10$ & 32.1 & 22.7 & 17.3 & 31.4 \\
\hline \multirow[t]{2}{*}{$40 \times 40$} & $1 \leq \mathrm{DAP}<10$ & 1517.5 & 186.0 & 155.5 & 19.6 \\
\hline & $\geq 10$ & 134.3 & 58.3 & 46.8 & 24.6 \\
\hline \multicolumn{6}{|c|}{ Belmira } \\
\hline \multirow[t]{2}{*}{$10 \times 10$} & $1 \leq \mathrm{DAP}<10$ & 32.8 & 27.2 & 13.3 & 104.4 \\
\hline & $\geq 10$ & 7.4 & 6.7 & 3.4 & 99.2 \\
\hline \multirow[t]{2}{*}{$20 \times 20$} & $1 \leq \mathrm{DAP}<10$ & 131.0 & 38.2 & 28.0 & 36.5 \\
\hline & $\geq 10$ & 29.5 & 10.3 & 7.8 & 32.1 \\
\hline \multirow[t]{2}{*}{$40 \times 40$} & $1 \leq \mathrm{DAP}<10$ & 524.5 & 59.0 & 46.5 & 26.9 \\
\hline & $\geq 10$ & 125.625 & 21.5 & 16.5 & 30.5 \\
\hline
\end{tabular}

especialmente entre Anorí y Belmira. Entre Anorí y Angelópolis la diversidad relativa fue casi constante a partir de aproximadamente 1 000 individuos, siendo la diversidad en Anorí 1.18 veces mayor que en Angelópolis; entre Belmira y las otras dos regiones, la diversidad relativa muestra la tendencia a seguir en aumento, siendo Belmira, en 2500 individuos, 5.25 y 4.41 veces menos diversa que los bosques de Anorí y Angelópolis, respectivamente (Fig. 5).

\section{DISCUSIÓN}

Este estudio tuvo como principal objetivo entender la variación de la diversidad y riqueza de especies arbóreas en un gradiente altitudinal, con el fin de probar las generalizaciones reportadas por investigaciones previas en bosques tropicales de tierras bajas (Condit et al. 1996). En este sentido, vale la pena mencionar que aunque los tres sitios aquí considerados no están evolutivamente aislados como los 

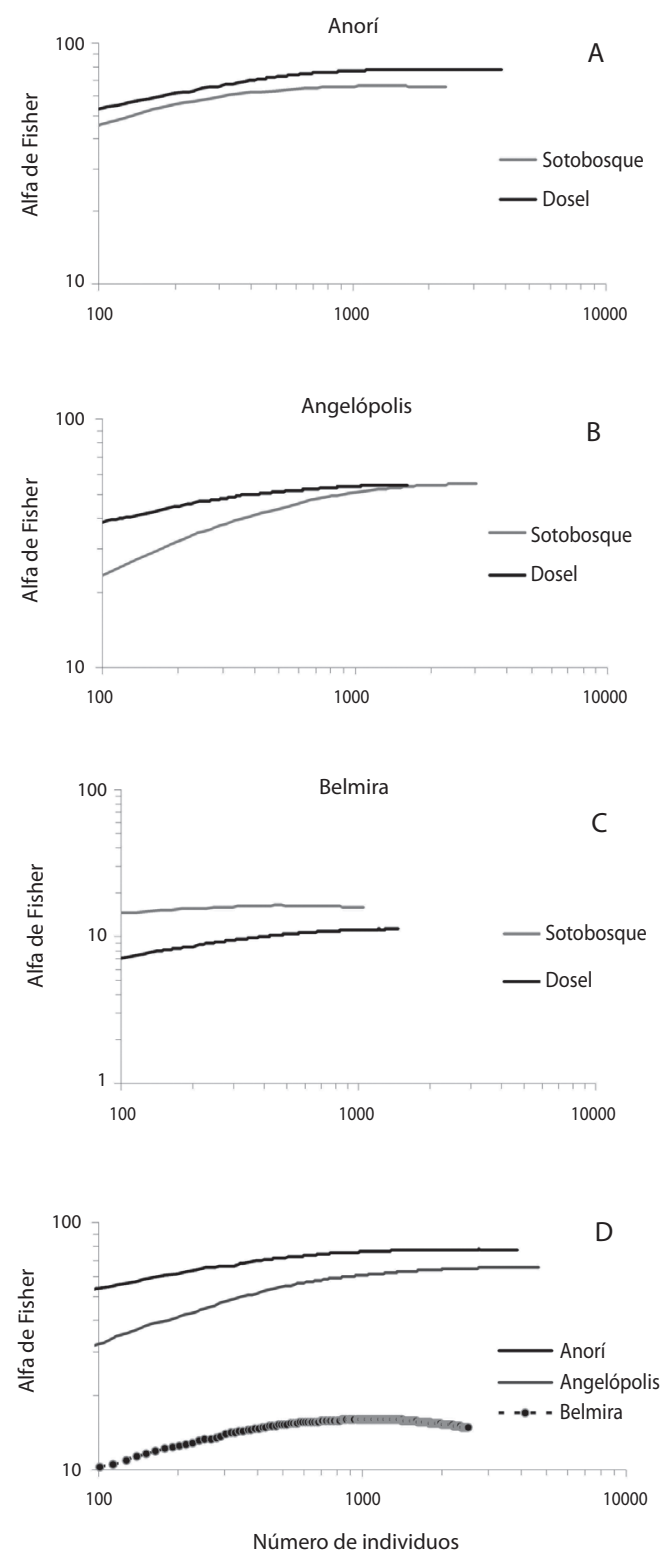

Fig. 4. Variación del índice de diversidad alfa de Fisher con el número de individuos por categorías de tamaño (A, $\mathrm{B}$ y C) y con los datos en conjunto para cada una de las regiones (D). En A, B y C, los puntos en gris representan el valor del alfa de Fisher para las especies del sotobosque y los puntos en negro representan las del dosel.

Fig 4. Fisher's alpha diversity index variation taking the individual number by size class (A, B, C) and the grouping data of each region (D). The grey dots show the Fisher's alpha diversity value for the understory and the black dots show the canopy values. mencionados en el estudio anterior (distancia geográfica entre 50 y $110 \mathrm{~km}$ ), su similitud florística es relativamente baja (índice de Jaccard, con base en datos de presencia ausencia, varía entre $5.4 \%$ y $11 \%$ entre regiones, López \& Duque en prep.) y su conectividad física debido a la fragmentación casi nula. Esta condición permite poner a prueba las generalizaciones emitidas acerca de los patrones de acumulación de especies y los mecanismos que la determinan en bosques tropicales a escala local (Condit et al. 1996, 1998, 2005).

Tasas de acumulación de especies en bosques de alta montaña: Es importante destacar que únicamente la riqueza en los bosques de Belmira presentó tendencia a la asíntota alrededor de dos hectáreas o 2000 individuos. Se concluye, por tanto, que los bosques alto Andinos en la región de Belmira, localizados en el límite arbóreo y caracterizados por su baja diversidad relativa, parecen poseer comunidades bien definidas en áreas de bosque entre 2 y 3 hectáreas (Gentry 1988, Tuomisto et al. 1995). En contraste, la diversidad y riqueza encontrada en Anorí y Angelópolis fueron similares, e incluso mayores, a la reportada para bosques de tierras bajas (Condit et al. 2004). Lo anterior se mantiene aún considerando que el área muestreada en muchas de dichas parcelas -entre 25 y 50 hectáreas- fueron superiores a las consideradas en este estudio. Esta última condición se hace relevante debido a que los sitios aquí estudiados se encuentran localizados a una altitud mayor de $1500 \mathrm{~m}$, límite superior para lo que se puede considerar una alta diversidad, de acuerdo a lo planteado por Gentry (1995).

En Belmira, los valores de $\mathrm{z}$ fueron menores a $0.1(z=0.05)$, valor promedio reportado en tierras bajas (Condit et al. 1996), donde el tamaño de muestra fue mucho mayor al considerado en este estudio (50 hectáreas). Este resultado sugiere dos posibles escenarios: 1) La tasa de acumulación de especies en Belmira, aunque baja, se mantiene constante o incrementa levemente con el aumento de la intensidad de muestreo, soportando las suposiciones de la 
CUADRO 2

Índice de alfa de Fisher para cada región

TABLE 2

Fisher's alpha diversity index for each region

\begin{tabular}{|c|c|c|c|c|c|}
\hline & Región & $\alpha$ Fisher Promedio \pm DS* & $\alpha(1000 \text { ind. })^{* *}$ & Parcela & $\alpha$ Fisher*** \\
\hline \multirow{2}{*}{\multicolumn{2}{|c|}{ Anorí }} & \multirow{2}{*}{$69.825 \pm 1.52$} & \multirow{2}{*}{75.88} & A & 68.75 \\
\hline & & & & B & 70.9 \\
\hline \multirow{2}{*}{\multicolumn{2}{|c|}{ Belmira }} & \multirow{2}{*}{$11.87 \pm 1.57$} & \multirow{2}{*}{15.95} & $\mathrm{C}$ & 12.98 \\
\hline & & & & $\mathrm{D}$ & 10.76 \\
\hline \multirow{2}{*}{\multicolumn{2}{|c|}{ Angelópolis }} & \multirow{2}{*}{$53.535 \pm 0.53$} & \multirow{2}{*}{61.15} & $\mathrm{E}$ & 53.91 \\
\hline & & & & $\mathrm{F}$ & 53.16 \\
\hline & Nariño & $31.45 \pm 2.24$ & $* * * *$ & La Planada & 29.31 \\
\hline *: & \multirow{3}{*}{\multicolumn{5}{|c|}{$\begin{array}{l}\text { Valor promedio por hectárea } \pm \text { DS: desviación estándar. Para Anorí, Angelópolis y Belmira n=2 y para La Planada } \\
\mathrm{n}=25 \text { (Samper \& Vallejo 2007). } \\
\alpha(1000 \text { ind.)Valor del índice alfa de Fisher para } 1000 \text { individuos en cada región. } \\
\text { Valor del índice alfa de Fisher en cada hectárea (A, B, C, D, E y F) y para } 25 \text { hectáreas en la parcela La Planada. } \\
\text { Dato no disponible. }\end{array}$}} \\
\hline$* *:$ & & & & & \\
\hline $\begin{array}{l}* * *: \\
* * * *:\end{array}$ & & & & & \\
\hline$*:$ & \multicolumn{5}{|c|}{$\begin{array}{l}\text { Hectare average value } \pm \text { DS: standard deviation. For Anorí, Angelópolis and Belmira n=2, La Planada n=25 (Samper } \\
\text { \& Vallejo 2007). }\end{array}$} \\
\hline **: & \multicolumn{5}{|c|}{$\alpha$ (1000 ind.) Fisher's alpha diversity index value for 1000 individuals in each region. } \\
\hline ***: & \multicolumn{5}{|c|}{$\begin{array}{l}\text { Fisher's alpha diversity index value for each hectare (plots A, B, C, D, E and F) and for } 25 \text { hectares in La Planada } \\
\text { plot. }\end{array}$} \\
\hline
\end{tabular}

deriva ecológica (Condit et al. 1996, Hubbell 2001). 2) El incremento de especies sigue disminuyendo con el aumento en la intensidad de muestreo, lo cual concuerda con la actual tendencia de $z$, corroborando el carácter discreto de las comunidades arbóreas en este tipo de bosque (Tuomisto et al. 1995) y en concordancia con los avances teóricos recientes que develan la tendencia a cero para el valor de $\mathrm{z}$ (Harte et al. 2009). En este tipo de bosque, donde se reportó una alta dominancia de $Q$. humboldtii en Belmira, la tendencia a la estabilización de $z$ en las tasas de cambio en las especies del dosel, podría explicarse por un incremento continuo de especies raras en los individuos de mayor tamaño del bosque. Un caso similar al aquí expuesto se reporta en bosques de tierras bajas como los de Ituri (R.D. del Congo), los cuales mostraron una alta dominancia de muy pocas especies y una diversidad inicial mucho menor en comparación con otros bosques cuando se empleó un tamaño de muestra pequeño (1 a 5 hectáreas); sin embargo, en Ituri, cuando se comparó la riqueza a tamaños de muestra más grandes (50 hectáreas), el número final de especies fue mayor que la de otros bosques que se suponían más ricos y diversos como los de Barro Colorado (Panamá), Sinharaja (Sri Lanka) y Huai Khae Khaeng (Tailandia) (Condit et al. 2004). Esto llama a la precaución para la interpretación de los resultados aquí obtenidos en términos de la riqueza de especies y el tamaño de las comunidades, dado el aún cuestionable tamaño total de la muestra (Condit et al. 1998, 2005).

En Anorí y Angelópolis, en contraste, la tasa de acumulación de especies sólo alcanzo valores mínimos de $z$ cercanos a 0.25 , similares a los reportados en otros estudios de curvas especie-área (Preston 1962). No obstante, dado 

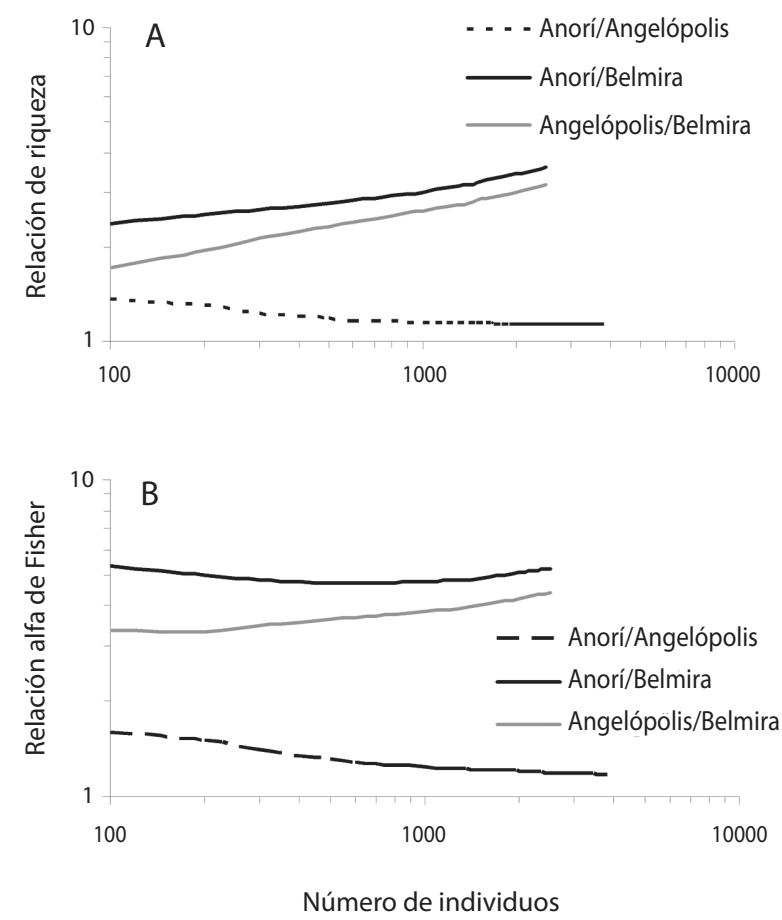

Fig. 5. Variación de la relación de riqueza (arriba) y diversidad (abajo) con el número de individuos. La diversidad fue medida con el índice alfa de Fisher.

Fig. 5. The ratio species numbers (above) and diversity (below) with the individual number. The Diversity was measured using the Fisher's alpha index.

que la tendencia de este valor es aún decreciente, se hace difícil inferir acerca del valor final esperado (a la asíntota o a $z=0$ ), con base en el actual tamaño de muestra. Este último resultado pone de manifiesto el efecto del submuestreo de la diversidad local en la mayoría de los bosques tropicales cuando el tamaño de muestra es relativamente pequeño (Condit et al. 2005), aumentando artificiosamente el número de especies raras e incrementado a su vez continua y constantemente la acumulación de especies en el espacio geográfico.

Curvas especies-área y especies-individuos como herramientas para la identificación de los determinantes de ensamblajes de especies: Las curvas especies-área fueron altamente sensibles al tamaño de los individuos considerados y, por ello, al tamaño de muestra. Es decir, mientras más pequeña la muestra, mayor es la sobre-estimación de las diferencias entre clases de tamaño. Esto se confirma por la tendencia de las curvas por tamaño para el dosel y el sotobosque, que tienden a converger a medida que incrementa el tamaño de muestra. Se concluye entonces que en bosques más diversos como los de Anorí y Angelópolis, se requieren tamaños de muestra muy grandes (mayores a 10000 individuos) para poder hacer comparaciones objetivas con base en la riqueza de especies, tal como se ha demostrado en bosques tropicales de tierras bajas (Condit et al. 1998, 2004, 2005).

Este trabajo coincide con otros estudios que concluyen que las curvas especies-individuos son la forma más apropiada para medir 
y comparar la diversidad alfa a lo largo de gradientes ambientales, independientemente del tamaño de los individuos considerado en los inventarios (Condit et al. 1996, Gotelli \& Colwell 2001). De acuerdo con nuestros resultados, lo anterior aplica para los estudios de diversidad en gradientes altitudinales donde se asume un cambio continuo en el número de especies. En Anorí y Angelópolis, el hecho de que las muestras de árboles del dosel fueron prácticamente idénticas a las del sotobosque, define las primeras esencialmente como muestras florísticas aleatorias de las más pequeñas (Condit et al. 1996). En Belmira en cambio, el patrón observado fue similar al reportado para el área, con una mayor diversidad en el sotobosque, lo cual denota un cambio en los patrones de dominancia y distribución de las especies entre el dosel y el sotobosque.

El número de especies observado fue sistemáticamente sobre-estimado por el valor esperado de una muestra aleatoria de individuos según lo propuesto por Hurlbert (1971), aunque de forma variable entre las regiones. A escalas finas $(10 \mathrm{mx} 10 \mathrm{~m})$, siempre fue mayor la sobre estimación y por tanto el gregarismo en el sotobosque que en el dosel lo cual propone a la limitación en dispersión (Hubbell 2001) como el principal mecanismo que determina los ensamblajes de especies a esta escala de análisis. Sin embargo, a tamaños de $40 \mathrm{~m} x$ $40 \mathrm{~m}$ la tendencia se invirtió, lo que sugiere que ha mayores escalas la denso-dependencia empieza ganar relevancia determinando los patrones de composición de especies (Janzen 1970, Connell 1971, Condit et al. 1994, Harms et al. 2000). Aunque, y de acuerdo con la curva de especies-individuos, en Angelópolis, no se afecta sensiblemente la abundancia total de las especies. En contraste, en Belmira se evidencia un efecto sobre el patrón de abundancia total y relativa de las especies comparativo entre dosel y sotobosque (Condit et al. 1996). Esto sugiere un patrón de agrupamiento a escalas finas extremadamente alto, lo que se traduce en un modelo de dominancia de pocas especies. Lo anterior se confirma por la marcada dominancia encontrada de la especie del dosel
Q. humboldtii, la cual representó cerca del 56\% de los individuos; en contraste, la especie de mayor dominancia en el sotobosque alcanzó el $11 \%$ de los individuos. Este resultado fue totalmente opuesto a lo encontrado en tierras bajas para los bosques de Barro Colorado (Panamá) y Pasoh (Malasia), donde la mayor desviación con respecto a la riqueza de especies observada se dio en las especies del sotobosque. En cambio, en Anorí no se presentó evidencia de denso-dependencia en los patrones de riqueza de especies lo cual sugiere una distribución cuasi aleatoria de las especies del dosel y sotobosque (menor grado de gregarismo), similar a lo reportado para los bosques de Mudumalai en la India (Condit et al. 1996).

El alfa de Fisher y sus ventajas en estudios comparativos: Los valores del índice alfa de Fisher variaron muy poco a partir de un número fijo de individuos en los tres bosques estudiados, lo cual confirma su utilidad en la predicción del número de especies. Esto se debe fundamentalmente a su independencia con respecto al tamaño de muestra cuando se incluyen por lo menos 1000 individuos (Condit et al. 2004). Resultados similares fueron encontrados en bosques de tierras bajas (Condit et al. 1996), corroborando la eficiencia de éste índice en el estudio de la diversidad en bosques tropicales. El hecho de que las curvas tuvieran una forma similar y no se interceptaran entre sí, denota la capacidad del índice para describir la relación entre el número de individuos y el número de especies. El alfa de Fisher ha mostrado ser robusto aún en casos de desviaciones de la abundancia total por especies no típicas de la serie logarítmica (mas especies abundantes y menos raras de lo esperado), lo cual concuerda con lo encontrado en Belmira (Condit et al. 2004).

Sin embargo, la tendencia a la asíntota y las bajas tasas decrecientes de $z$ en los bosques de Belmira, sugieren que el índice tendería eventualmente a decrecer con mayores tamaños de muestra. De esta manera, se debe tener precaución especialmente con las especies del sotobosque en este tipo de bosque altoandino, 
cuando se desea extrapolar la riqueza usando este índice.

No obstante, el alfa de Fisher aparece como una alternativa muy eficiente para entender la magnitud de las diferencias cuantitativas en diversidad entre regiones o tipos de bosque en gradientes altitudinales, especialmente cuando el tamaño de la muestra es relativamente pequeño. En nuestro caso, la razón esperada de la diferencia comparativa de la riqueza si se tuvieran tamaños de muestra más grandes, parece haber sido bien develada por el alfa de Fisher. Esto permite comparaciones más objetivas incluso con los bosques de tierras bajas, que las hasta ahora asumidas con base en la riqueza de especies (Gentry 1995).

Consideraciones finales: Varias conclusiones importantes relacionadas con el estudio de la diversidad y riqueza de especies en bosques tropicales, asociadas con gradientes altitudinales, emergen como resultado de este estudio de caso. En primer lugar, cuando se tienen tamaños de muestra relativamente pequeños, las curvas especies-individuos son una forma más acertada de comparar la diversidad entre regiones y tipos de bosque que las curvas especies-área. Segundo, en bosques por encima de los $2100 \mathrm{msnm}$, la denso-dependencia jugaría un rol importante en la estructuración de las comunidades; de esta manera, las generalizaciones con base en la deriva ecológica son altamente debatibles. Sin embargo, es necesario aclarar que aunque algunos patrones observados en Angelópolis y Belmira fueron totalmente opuestos a los reportados para tierras bajas, se requiere de estudios más amplios para poder confirmar las conclusiones de éste estudio en cuanto a la existencia de una relación positiva entre el incremento de procesos denso-dependientes y la altitud en bosques tropicales. Por último, se concluye de este estudio que el alfa de Fisher es una herramienta muy eficaz para estimar la magnitud de las diferencias esperadas en términos de la riqueza entre regiones con tamaños de muestra más grandes, con base en un número limitado de individuos.

\section{AGRADECIMENTOS}

Queremos agradecer a las siguientes personas e instituciones que de una u otra manera contribuyeron a la elaboración del presente trabajo: A Juan Lázaro Toro quien contribuyó enormemente al desarrollo logístico. A Jorge Pérez -director del Herbario Gabriel Gutiérrez V. (MEDEL)-, Álvaro Idárraga, Ricardo Callejas, Heriberto Marín, Adriana Rivas, Dino Tuberquia y Felipe Cardona -Herbario Universidad de Antioquia (HUA)-, quienes colaboraron con la determinación del material vegetal. A la Corporación Autónoma Regional del Centro y Norte de Antioquia (CORANTIOQUIA) y a la Dirección de Investigación de la Universidad Nacional de Colombia, Sede Medellín (DIME) por la financiación que hizo posible la realización del estudio. Se recibió el apoyo logístico del Mountain Research Initiative (MRI), que hace parte de los productos asociados con la red de parcelas Cordillera Forest Dynamics Network (CORFOR, http//www.corfor.com).

\section{RESUMEN}

En el presente trabajo se analizaron los patrones de diversidad alfa en tres áreas protegidas de bosque con base en información florística proveniente de seis parcelas permanentes de 1 ha -dos en cada área protegida-. Las regiones de estudio están localizadas a lo largo de un gradiente altitudinal, en la parte norte de la cordillera central de los Andes, Colombia. Para los análisis, se generaron dos categorías de acuerdo al tamaño de los árboles: dosel (diámetro a la altura del pecho mayor o igual a $10 \mathrm{~cm}$ ) y sotobosque (diámetro a la altura del pecho mayor o igual a un $\mathrm{cm}$ y menor a $10 \mathrm{~cm}$ ). Las curvas especies-área mostraron diferencias en riqueza para las dos categorías de tamaño en las tres regiones. Las curvas especies-individuos, en cambio, fueron independientes con respecto al tamaño de los individuos, excepto en la región de Belmira. La diversidad, medida con el índice alfa de Fisher, mostró una fuerte tendencia a la estabilización en muestras mayores a 1000 individuos. $\mathrm{Ni}$ las curvas de especies-área ni las de especies-individuos fueron asintóticas; sin embargo, la tasa de acumulación de especies $(z)$ en Belmira alcanzó valores muy bajos (0.05) en comparación con Anorí y Angelópolis (0.25 en cada caso). El índice alfa de Fisher permitió además entender la magnitud de las diferencias relativas en diversidad entre regiones o tipos de bosque en gradientes altitudinales, aun cuando el tamaño de la muestra fue relativamente pequeño. Los resultados de este estudio soportan la limitación en 
dispersión como el principal mecanismo en estructurar las comunidades de especies a escalas muy finas, y un incremento continuo de procesos denso-dependientes en determinar la estructura de la comunidad en escalas espaciales más grandes. Igualmente, los resultados de este estudio proponen que la denso-dependencia tiende a aumentar positivamente con la altitud.

Palabras clave: bosques montanos, Andes, diversidad alfa, curvas especies-área, curvas especies-individuos, alfa de Fisher, limitación es dispersión, denso-dependencia, Colombia.

\section{REFERENCIAS}

Borda-de-Agua, L., S.P. Hubbell \& M. McAllister. 2002. Species-area curves, diversity indices, and species abundance distributions: A multifractal analysis. Am. Nat. 159: 138-155.

Boyle, B. L. 1996. Changes on altitudinal and latitudinal gradients in Neotropical montane forests. A dissertation Graduate School of Arts and Sciences. Tesis de doctorado, Universidad de Washington, Washington D.C., EEUU.

Colwell, R.K. 2005. Estimate S: Statistical estimation of species richness and shared species from samples. Version 7.5.1 User's Guide and application. University Connecticut, Storrs, Connecticut, EEUU (Julio 6, 2007, http://purl.oclc.org/estimates).

Condit, R., S.P. Hubbell \& R.B. Foster. 1994. Densitydependence in two understory tree species in a neotropical forest. Ecology 75: 671-680.

Condit, R., S.P. Hubbell, J.V. Lafrankie, R. Sukumar, N. Manokaran, R. Foster \& P. Ashton. 1996. SpeciesArea and Species-Individual relationships for tropical trees: A comparison of three 50 ha plots. J. Ecol. 4: 549-562.

Condit, R., R.B. Foster, S.P. Hubbell, R. Sukumar, E.G. Leigh, N. Manokaran, S. Loo de Lao, J.V. LaFrankie \& P.S. Ashton. 1998. Assessing forest diversity on small plots: calibration using species-individual curves from 50 ha plots, p. 247-268. In F. Dallmeier \& J.A. Comiskey. Forest Biodiversity Research, Monitoring and Modeling. MAB Series Vol. 20, UNESCO and Parthenon Publishing Group, Paris, Francia and NuevaYork, EEUU.

Condit, R., E.J. Leigh, S. Loo de Lao, S. Ashton, N. Brokaw, V.L. Nicholas, R. Bunyavejchwin, G.B. Chuyong, L. Co, H.S. Dattaraja, S. Davies, S. Esufali, C.E.N. Ewango, R. Foster, N. Gunatilleke, S. Gunatilleke, T. Hart, C. Hernandez, S.P. Hubbell, A. Itoh,
R. John, M. Kanzaki, D. Kenfack, S. Kiratiprayoon, J. La Frankie, H.S. Lee, I. Liengola, J.R. akana, N. Manokaran, H. Navarette, T. Ohkugo, R. Perez, N. Pongpattananurak, C. Samper, R. Sukumar, I.F. Fun, H.S. Sureh, S. Tan, D. Thomas, J. Thompson, M. Vallejo, G. Villa, R. Valencia, T. Yamakura \& J.K. Zimmerman. 2004. Species-area relationships and diversity measures in the forest dynamics plots, p.7989. In E. Losos \& E.G. Leigh. Tropical forest diversity and dynamism: Findings from a large-scale plot network. Chicago Press, Chicago, Illinois, EEUU.

Condit, R., P.S. Ashton, H. Balslev, S. Bunyavejchewin, G. Cuyong, L. Co, H.S. Dattaraja, S. Davies, S. Esufali, C.E.N. Ewango, R. Foster, S. Gunatilleke, N. Gunatilleke, C. Hernandez, S.P. Hubbell, R. John, D. Kenfack, S. Kiratiprayoon, P. Hall, L. Hua Seng, A. Itoh, I. LaFrankie, S. Liengola, E. Lao, E. Losos, J. Magard, N. Makana, J. Manokaran, H. Navarrete, S. Mohammed Nur, R. Sukumar, J. Svenning, D. Tan, S. Thomas, J. Thomspon, M. Vallejo, G. Villa, R. Valencia, T. Yamakura \& J. Zimmerman. 2005. Tropical tree $\alpha$-diversity: results from a worldwide network of large plots. Bio. Skr. 55: 565-582.

Connell, J.H. 1971. On the role of enemies in preventing competitive exclusion in some marine animals and in rain forest trees, p. 298-312. In P.J. den Boer \& G.R. Gradwell. Dynamics of Populations. Center for Agricultural Publication and Documentation. Wageningen, Güeldres, Holanda.

Duque, A., W. López, F. Moreno \& J.L. Toro. 2008. Variabilidad estructural y ambiental en remanentes de bosques andinos, p.17-33. In J.D. León. Ecosistemas de bosques andinos. Universidad Nacional, Medellín, Antioquia, Colombia.

Gentry, A. 1995. Patterns of diversity and floristic composition in Neotropical Montane Forests, p. 103-126. In S.Churchill, H. Balslev, E. Forero \& J. Luteyn. Biodiversity and Conservation of Neotropical Montane Forests. The New York Botanical Garden, Nueva York, Nueva York, EEUU.

Gentry, A.H. 1988. Changes in plant community diversity and floristic composition on environmental and geographical gradients. Ann. Mo. Bot. Gard. 75: 1-34.

Gotelli, N.J. \& R. Colwell. 2001. Quantifying biodiversity: procedures and pitfalls in the measurement and comparison of species richness. Ecol. Lett. 4: 379-391.

Gutiérrez-C, P.D.A. 2002. Plan de Manejo Ambiental para el Área de Manejo Especial La Forzosa. Corporación Autonoma Regional del Centro de Antioquia -Corantioquia- Medellín, Antioquia, Colombia. 
Harms, K.E., S.J. Wright, O. Calderón, A. Hernández \& E.A. Herre. 2000. Pervasive density-dependent recruitment enhances seedling diversity in a tropical forest. Nature 404: 493-495.

Harte, J., A. Kinzig \& J. Green. 1999. Self-Similarity in the Distribution and Abundance of Species. Science 284: 334-336.

Harte, J., T. Zillio, E. Conlisk \& A. Smith. 2008. Maximum entropy and the state-variable approach to macroecology. Ecology 89: 2700-2711.

Harte, J., A.B. Smith \& D. Storch. 2009. Biodiversity scales from plots to biomes with a universal species-area curve. Ecol. Lett. 12: 789-797.

He, F. \& P. Legendre. 1996. On species-area relations. Am. Nat. 148: 719-737.

He, F. \& P. Legendre. 2002. Species diversity patterns derived from species-area models. Ecology 83: 11851198 .

Hemp, A. 2005. Continuum or zonation? Altitudinal gradients in the forest vegetation of Mt. Kilimanjaro. Plant. Ecol. 184: 27-42.

Holdridge, L. 1996. Ecología basada en Zonas de Vida Instituto Interamericano de Cooperación para la Agricultura, San José, San José, Costa Rica.

Hubbell, S.P. 2001. The unified neutral theory of biodiversity and biogeography. Monographs in Population Biology 32. Princeton University Press, Princeton, Nueva Jersey, EEUU.

Hurlbert S.H. 1971. The non-concept of species diversity: A critique and alternative parameters. Ecology 52: 577-586.

IUCN. 2008. Red List of Threatened Species. The International Union for Concervation of Nature. Fontainebleau, Francia (también disponible en línea: www. iucnredlist.org).

Janzen, D.H. 1970. Herbivores and the number of tree species in tropical forests. The Am. Nat. 104: 501-528.

Lieberman, D., M. Lieberman, R. Peralta \& G. Hartshorn. 1996. Tropical forest structure and composition on a large-scale altitudinal gradient in Costa Rica. J. Trop. Ecol. 84: 137-152.

López, W. 2008. Diversidad y tasas de recambio florístico en remanentes de bosques montanos en el Norte de la Cordillera Central colombiana. Tesis de maestría, Universidad Nacional, Medellín, Antioquia, Colombia.

Mabberley, D.J. 1989. The Plant-book. Universidad de Cambridge, Cambridge, Cambridgeshire, Reino Unido.

Mac Arthur, R. \& E.O. Wilson. 1967. The Theory of Island Biogeography. Princeton University Press, Princeton, Nueva Jersey, EEUU.

Magurran, A. 1988. Ecological diversity and its measurement. Princeton University, Princeton, Nueva Jersey, EEUU.

Plotkin, J.B., M.D. Potts, D.W. Yu, S. Bunyavejchewin, R. Condit, R. Foster, S. Hubbell, J. LaFrankie, N. Manokaran, H. Lee, R. Sukumar, M.A. Nowak \& P.S. Ashton. 2000. Predicting species diversity in tropical forests. PNAS 97: 10850-10854.

Preston, F.W. 1962. The canonical distribution of commonness and rarity. Ecology 43: 185-215.

Rodríguez N., D. Armenteras, M. Morales \& M. Romero. 2004. Ecosistemas de los Andes Colombianos. Instituto de Investigación de Recursos Biológicos Alexander von Humboldt, Bogotá, Cundinamarca, Colombia.

Rosenzweig, M.L. 1995. Species diversity in space and time. Universidad de Cambridge, Cambridge, Cambridgeshire, Reino Unido.

Samper, C. \& M.I. Vallejo. 2007. Estructura y dinámica de poblaciones de plantas en un bosque andino. Rev. Acad. Colomb. Cienc. 31: 57-68.

Tuomisto, H., K. Ruokolainen, R. Kalliola, A. Linna, W. Danjoy \& Z. Rodríguez. 1995. Dissecting Amazonian Biodiversity. Science 269: 63-66.

Zillio, T., \& R. Condit. 2007. The impact of neutrality, niche differentiation and species input on diversity and abundance distributions. Oikos 116: 931-940. 